\title{
BMJ
}

\section{Inadequate reporting of research ethics review and informed consent in cluster randomised trials: review of random sample of published trials}

\author{
Monica Taljaard, scientist, ${ }^{1}$ Andrew D McRae, research director, ${ }^{2}$ Charles Weijer, professor, ${ }^{3}$ Carol Bennett, \\ research coordinator, ${ }^{1}$ Stephanie Dixon, postdoctoral fellow, ${ }^{4}$ Julia Taleban, PhD candidate, ${ }^{4}$ Zoe Skea, \\ research fellow, ${ }^{5}$ Martin P Eccles, professor, ${ }^{6}$ Jamie C Brehaut, scientist, ${ }^{1}$ Allan Donner, professor, ${ }^{4}$ Raphael \\ Saginur, professor, ${ }^{7}$ Robert F Boruch, professor, ${ }^{8}$ Jeremy M Grimshaw, senior scientist ${ }^{1}$
}

${ }^{1}$ Clinical Epidemiology Program, Ottawa Hospital Research Institute, Ottawa Hospital, Civic Campus, 1053 Carling Avenue, Ottawa, ON Canada K1Y 4E9

${ }^{2}$ Division of Emergency Medicine, University of Calgary, Foothills Medical Centre, Calgary, AB, Canada

${ }^{3}$ Philosophy and Medicine, Rotman Institute of Philosophy, Department of Philosophy,

University of Western Ontario, London, ON, Canada

${ }^{4}$ Department of Epidemiology and Biostatistics, Schulich School of Medicine and Density, University of Western Ontario, London, ON, Canada

${ }^{5}$ Health Services Research Unit, University of Aberdeen,

Foresterhill, Aberdeen AB25 2ZD, UK

${ }^{6}$ Clinical Effectiveness, Institute of Health and Society, Newcastle

University, Newcastle upon Tyne NE2 4AX, UK

${ }^{7}$ Medicine, Ottawa Hospital, Ottawa ON, Canada

${ }^{8}$ Education and Statistics, Graduate School of Education,

University of Pennsylvania, Philadelphia, PA 19104, USA Correspondence to: $M$ Taljaard mtaljaard@ohri.ca

Cite this as: $B M J$ 2011;342:d2496 doi:10.1136/bmj.d2496

\section{ABSTRACT}

Objectives To investigate the extent to which authors of cluster randomised trials adhered to two basic requirements of the World Medical Association's Declaration of Helsinki and the International Committee of Medical Journal Editors' uniform requirements for manuscripts (namely, reporting of research ethics review and informed consent), to determine whether the adequacy of reporting has improved over time, and to identify characteristics of cluster randomised trials associated with reporting of ethics practices.

Design Review of a random sample of published cluster randomised trials from an electronic search in Medline.

Setting Cluster randomised trials in health research published in English language journals from 2000 to 2008.

Study sample 300 cluster randomised trials published in 150 journals.

Results 77 (26\%, 95\% confidence interval $21 \%$ to $31 \%$ ) trials failed to report ethics review. The proportion reporting ethics review increased significantly over time $(P<0.001)$. Trials with data collection interventions at the individual level were more likely to report ethics review than were trials that used routine data sources only $(79 \%$ $(\mathrm{n}=151) \vee 55 \%$ (23); $\mathrm{P}=0.008)$. Trials that accounted for clustering in the design and analysis were more likely to report ethics review. The median impact factor of the journal of publication was higher for trials that reported ethics review (3.4 v 2.3; P<0.001). 93 (31\%, 26\% to 36\%) trials failed to report consent. Reporting of consent increased significantly over time ( $\mathrm{P}<0.001)$. Trials with interventions targeting participants at the individual level were more likely to report consent than were trials with interventions targeting the cluster level (87\% (90) v 48\% (41); P<0.001). Trials with data collection interventions at the individual level were more likely to report consent than were those that used routine data sources only $(78 \%$ (146) v 29\% (11); P<0.001)

Conclusions Reporting of research ethics protections in cluster randomised trials is inadequate. In addition to research ethics approval, authors should report whether informed consent was sought, from whom consent was sought, and what consent was for.

\section{INTRODUCTION}

Cluster randomised trials are distinct from other randomised controlled trials in that the units of random assignment are intact clusters of entities such as entire medical practices, hospitals, schools, or communities, rather than the constituent individuals of these units themselves. ${ }^{1}$ The statistical implications of this hierarchical structure are well recognised, and an extensive literature exists to provide guidance for the appropriate design and analysis of these trials, but relatively little attention has been paid to the ethical implications. ${ }^{2-5}$ Obtaining informed consent, for example, is more complicated in cluster randomised trials, not only because groups of participants need to be randomised as a whole (often before identification of eligible participants within clusters) but also because the experimental treatment may need to be delivered to the cluster as a whole. The experimental treatment may be a medical therapy, health promotion campaign, or public health intervention targeting individual members of a cluster, or it may be an educational intervention targeting a health professional or a quality improvement intervention targeting the cluster organisation. Moreover, a "gatekeeper" or someone who is authorised to consent on behalf of a cluster without necessarily receiving the intervention him/herself may exist (for example, a school principal or the chief executive at a hospital). Furthermore, outcomes of interest may need to be collected from participants at the level of the individual (such as patients, students, or employees), the cluster (such as health professionals, school teachers, or shift supervisors), or both, or they may be available from routine data sources within each cluster (for example, patients' medical records, health insurance records). Althabe et al, for example, randomised 19 hospitals to receive a multifaceted behavioural intervention to promote implementation of evidence based obstetrical 
practices. ${ }^{6}$ The unit of randomisation was the hospital, the intervention was targeted at birth attendants in each hospital, and outcomes were observed in individual patients. In this trial, responsible authorities at each hospital, as well as birth attendants, provided written consent to participate in the trial; no consent was sought from patients, as outcomes were part of routine data collected at each hospital with no personal identifying information transmitted.

We report here one component of a project to study ethical challenges in health related cluster randomised trials. ${ }^{7}$ An in-depth analysis of the ethical challenges, together with preferred solutions, is presented elsewhere $^{8}$; here, we focus on the practice of the reporting of two key pieces of information relevant to a variety of these ethical requirements - namely, approval by a research ethics committee and informed consent. According to the World Medical Association's Declaration of Helsinki, research studies should be approved by an independent research ethics committee and, when appropriate, informed consent should be sought from study participants. ${ }^{9}$ Likewise, the UK Medical Research Council's guidelines for the conduct of cluster randomised trials require ethics review and voluntary informed consent from participants, where possible. ${ }^{10}$ The International Committee of Medical Journal Editors' (ICMJE's) uniform requirements for manuscripts submitted to biomedical journals requires that: "authors should indicate whether the procedures followed were in accordance with the ethical standards of the responsible committee on human experimentation (institutional and national) and with the Helsinki Declaration... If doubt exists whether the research was conducted in accordance with the Helsinki Declaration, the authors must explain the rationale for their approach and demonstrate that the institutional review

\author{
Medline search strategy to identify cluster randomised trials (sensitivity=90.1\%, \\ precision=18.4\%) \\ 1. randomized controlled trial.pt. \\ 2. animals/ \\ 3. humans/ \\ 4. 2 NOT (2 AND 3) \\ 5. 1 NOT 4 \\ 6. cluster\$ adj2 randomi\$.tw. \\ 7. ((communit\$ adj2 intervention\$) OR (communit\$ adj2 randomi\$)).tw. \\ 8. group $\$$ randomi $\$ . t w$. \\ 9. 6 OR 7 OR 8 \\ 10. intervention?.tw. \\ 11. cluster analysis/ \\ 12. health promotion/ \\ 13. program evaluation/ \\ 14. health education/ \\ 15. 10 OR 11 OR 12 OR 13 OR 14 \\ 16. 9 OR 15 \\ 17. 16 AND 5
}

body explicitly approved the doubtful aspects of the study." 11 Close to 900 journals now state that they have adopted the ICMJE's requirements.

The minimum requirements of clear reporting of ethics practices apply equally in cluster randomised trials. Donner and Klar proposed that: "As a first step in developing a well-accepted set of ethical principles and norms for cluster randomization trials, editors could require all articles describing results to report having institutional review board approval." They also recommend that: "When permission from key decision-makers associated with each cluster is needed for assigning interventions, some indication should be provided as to who these decision-makers are and how they were identified. Some information about the consent procedure administered to individual study participants should also be provided." 5

This study aimed to review the adequacy of reporting of these minimum ethical criteria in cluster randomised trials. On the basis of a representative sample of published cluster randomised trials, our primary objectives were to investigate the frequency of reporting of research ethics review and informed consent in cluster randomised trials in health research, investigate whether the rate of reporting has increased over time, and identify methodological characteristics associated with reporting these basic ethical requirements.

\section{METHODS}

Search strategy and article selection

We used an electronic search strategy (box) with a sensitivity of $90.1 \%$ and a precision of $18.4 \%$ to identify reports of cluster randomised trials in health research, published in English language journals between 2000 and 2008. ${ }^{12}$ We implemented the search strategy on 4 December 2008 in the Ovid Medline database from 1996 to the third week of November 2008. We assigned a computer generated uniform random number to each identified report. We sorted reports by random number and screened titles and abstracts of reports (as well as full text where necessary) to identify trials that met our eligibility criteria. For calibration purposes, two reviewers (MT and $\mathrm{CB}$ ) initially screened a training sample of 300 records. They discussed disagreements and calculated the $\kappa$ statistic to assess agreement in the identification of trials. If the $\mathrm{k}$ statistic did not reach 0.85 , both reviewers screened the next 300 records until the $\kappa$ statistic reached at least 0.85 . Thereafter, reviewers screened records separately until the target sample size of 300 eligible cluster randomised trials was reached. We used the sample size of 300 trials to restrict the width of a 95\% two sided confidence interval around the estimated proportion of trials reporting research ethics review or informed consent. The maximum width of $11.4 \%$ is obtained by using the most conservative estimate of observed proportion equal to $50 \%$.

\section{Inclusion and exclusion criteria}

We included an article if it was the main report of a cluster randomised trial. We excluded pilot studies, 
trial protocols, trials randomising households or dyads of different individuals, short communications or conference proceedings, trials with further allocation of individuals within clusters, quasi-randomised designs, secondary analyses of trials, and trials reporting only baseline findings.

\section{Data abstraction}

We developed data abstraction forms, pilot tested them, and then applied them to a sample of 21 cluster randomised trials for calibration of reviewers. All six reviewers (MT, ADMcR, CB, SD, JT, and ZS) independently abstracted these 21 trials. We identified differences and resolved them by discussion. Rotating pairs of reviewers then independently abstracted the remainder of the trials. After each set of 20 trials had been abstracted, discrepancies were reviewed within the pair and resolved by consensus. If differences could not be resolved, one reviewer (MT) was the arbitrator.

We abstracted information about ethics review procedures for the trials: whether or not approval was reported, whether the ethics committees were identified in the report, and, if so, how many committees were involved. If an author reported that a trial was exempt from ethics review, we recorded the reason for the exemption, if explained. We further extracted information about any informed consent procedures reported, distinguishing between consent from participants at the level of the cluster and the individual. We also recorded any other statements of consent-for example, from someone who could be considered a gatekeeper. If an author reported that a waiver of consent had been obtained from the research ethics committee, we recorded the reason for the waiver, if explained.

The data abstraction instrument also collected items on year of publication, country of study recruitment, type of cluster being randomised, number and size of clusters randomised, and study design and analysis. For each trial, we recorded whether any experimental interventions targeted participants at the individual level and at the cluster level. We also recorded the type of data collection activities that were used, specified as the use of routinely available data (such as administrative or medical records) or the use of any direct intervention on or interaction with participants for purposes of data collection, including physical examination, medical tests, or interactions such as surveys or interviews at both the individual level and the cluster level.

\section{Analysis}

We summarised results by using frequencies and percentages for categorical variables and medians and interquartile ranges for continuous or ordinal variables; we used asymptotic or exact methods to calculate $95 \%$ confidence intervals for proportions where appropriate. To test the significance of changes over time, we used the Cochran-Armitage test for trend. We tested associations between the reporting of ethical practices and characteristics of trials by using Pearson $\chi^{2}$ tests for categorical variables and Wilcoxon two sample tests for continuous variables. Characteristics of trials investigated included the level(s) at which interventions were offered, categorised as cluster level only, individual level only, or both individual and cluster level, as well as the types of data collection activities that were used, categorised as the use of

Table 1|Characteristics of cluster randomised trials included in review. Values are numbers (percentages) unless stated otherwise

\begin{tabular}{ll} 
Characteristic & Value $(n=300)$ \\
Publication year: & \\
\hline $2000-1$ & $44(15)$ \\
\hline $2002-3$ & $49(16)$ \\
\hline $2004-5$ & $87(29)$ \\
\hline $2006-7$ & $99(33)$ \\
\hline 2008 & $21(7)$
\end{tabular}

Journal impact factor $(n=294)$ :

\begin{tabular}{lc} 
Median (interquartile range) & $2.9(2.1-5.1)$ \\
\hline Range & $0.45-50.0$ \\
\hline
\end{tabular}

Country of study recruitment:

\begin{tabular}{lc}
\hline United States & $114(38)$ \\
\hline UK or Ireland & $50(17)$ \\
\hline Canada & $16(5)$ \\
\hline Australia or New Zealand & $16(5)$ \\
\hline Other & $104(35)$ \\
\hline
\end{tabular}

Country's level of development:

\begin{tabular}{lc} 
Advanced economies & $255(85)$ \\
\hline Emerging and developing economies & $45(15)$
\end{tabular}

Healthcare organisation or setting:

\begin{tabular}{lc}
\hline Medical practices or clinics & $81(27)$ \\
\hline Individual health professionals & $41(14)$ \\
\hline Hospitals, hospital units, hospital wards & $25(8)$ \\
\hline Nursing homes or wards & $16(5)$ \\
\hline $\begin{array}{l}\text { Residential areas (such as postal codes of } \\
\text { family practices) }\end{array}$ & $6(2)$
\end{tabular}

Non-healthcare organisation or setting:

\begin{tabular}{ll} 
Schools or classrooms & $66(22)$ \\
$\begin{array}{l}\text { Residential areas (such as villages, districts, } \\
\text { housing units) }\end{array}$ & $39(13)$ \\
\hline Worksites & $16(5)$ \\
\hline $\begin{array}{l}\text { Sports teams, clubs, churches, other social } \\
\text { groups }\end{array}$ & $10(3)$ \\
\hline
\end{tabular}

groups

No of clusters randomised $(n=285)$ :

Median (interquartile range) 21.0 (12-52)

Range $2-605$

Average cluster size $(n=271)$ :

Median (interquartile range) $33.9(12.5-88.5)$

Range

1.7-122 855

Target of interventions:

\begin{tabular}{lc}
\hline Cluster level only & $99(33)$ \\
\hline Individual level only & $105(35)$ \\
\hline Both cluster level and individual level & $96(32)$ \\
\hline Types of data collection interventions: & $192(64)$ \\
\hline Individual level only & $23(8)$ \\
\hline Cluster level only & $40(13)$ \\
\hline Both individual level and cluster level & $45(15)$ \\
\hline
\end{tabular}




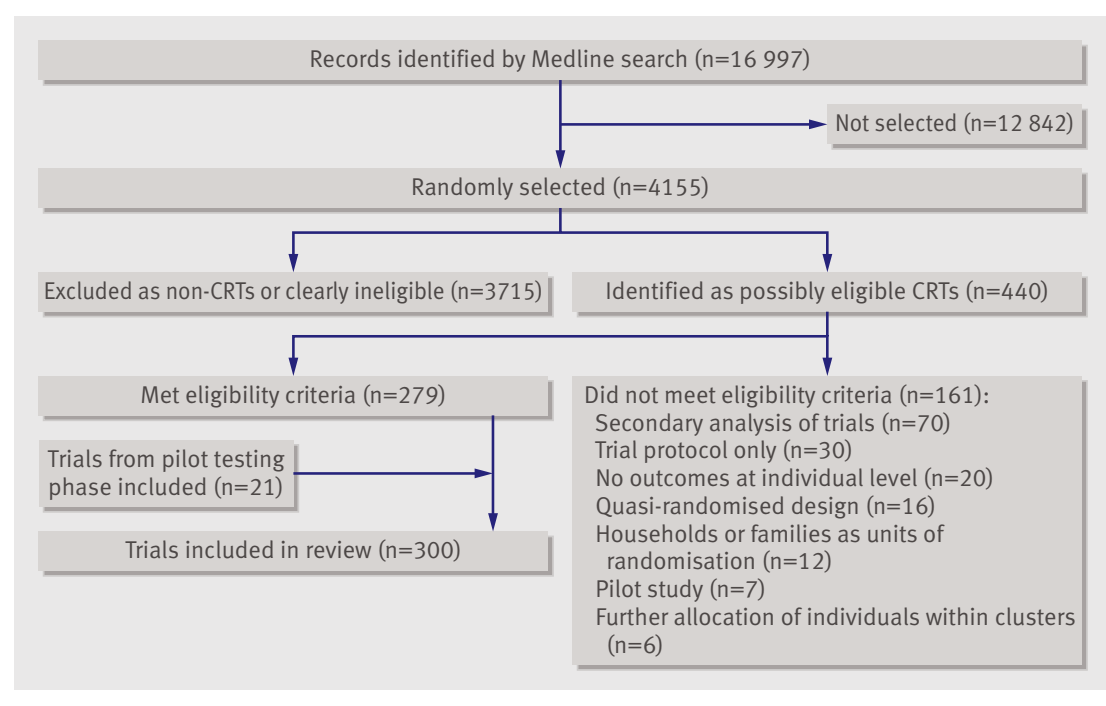

Identification of sample of 300 cluster randomised trials (CRTs) included in review

routinely available data only or data collection interventions administered to participants at the cluster level, the individual level, or both. We also investigated associations with the country where the study took place, as well as its level of development, classified as advanced economies versus emerging and developing economies according to the October 2008 edition of the world economic outlook database. ${ }^{13}$ We investigated associations with cluster type by grouping together trials in which the unit of randomisation or intervention was a healthcare provider or healthcare organisation, such as a primary care practice, hospital ward, or nursing home, and comparing them with trials involving non-healthcare organisations such as schools, worksites, or residential areas.

To investigate associations with methodological and reporting quality of trials, we classified each trial on the basis of whether two key methodological and reporting requirements for cluster randomised trials were metnamely, accounting for clustering during sample size calculation and accounting for clustering during analysis, as stipulated by the CONSORT statement extension to cluster randomised trials. ${ }^{14}$ Trials that fail to account for clustering in the design risk underestimating sample size requirements, and trials that fail to account for clustering in the analysis risk overstating the statistical significance of the findings. ${ }^{1}$ We classified a trial as meeting the sample size requirement if the sample size calculation was presented and clearly accounted for clustering (for example, by using the intracluster correlation coefficient, coefficient of variation, or cluster level summary statistics), and we classified a trial as meeting the analysis requirement if the method of analysis was reported and was clearly appropriate for the clustered design (for example, adjustment for the intracluster correlation coefficient, use of a mixed effects regression analysis, or use of cluster level summary statistics). We also investigated associations with journal impact factor, obtained from journal citation reports (ISI Web of Science, 2009). For journals with no ranking available, we used the journal impact ranking of the open access SMImago journal and country rank database, if available. ${ }^{15}$ The SMImago journal and country rank is calculated with a similar formula and is very strongly correlated with the journal citation report ranking. ${ }^{16}$ We used SAS v.9.1 for all analyses, with a level of significance set at $\alpha=0.01$. We selected a stringent level of significance because we were doing multiple tests of association.

\section{RESULTS}

We identified close to 17000 articles with the search strategy (figure). We reached the target sample size of 300 eligible cluster randomised trials after screening a random sample of 4155 records. Identification of trials as cluster randomised trials in titles or abstracts was poor, and in a large number of cases we had to download the full article to confirm whether it was a cluster randomised trial. The $\kappa$ statistic for agreement on inclusion of studies was 0.87 after three rounds of screening (the $\kappa$ statistics in the first two rounds were 0.75 and 0.83 ).

Table 1 shows the characteristics of the 300 trials included in the review. We included trials from 150 different journals (listed in web appendix). Four fifths of the studies were dispersed among 146 different journals, whereas four journals included approximately one fifth of the trials: $B M J$ (22 trials), JAMA (12 trials), Lancet (11 trials), and Preventive Medicine (11 trials). Slightly more than two thirds of reports in our sample were published during or after 2004, suggesting an increase in the use of cluster randomisation over time. The types of clusters randomised were diverse and included medical practices, nursing homes, individual health professionals, residential areas, schools, worksites, and social units such as sports teams, churches, or civic clubs.

Trials represented diverse types of interventions: the target of the intervention was exclusively at the indivi-

Table 2|Reporting of research ethics review in cluster randomised trials. Values are numbers (percentages) unless stated otherwise

\begin{tabular}{|c|c|c|}
\hline & Value $(n=300)$ & $\begin{array}{l}95 \% \text { confidence } \\
\text { interval of } \%\end{array}$ \\
\hline \multicolumn{3}{|c|}{ Research ethics review reported? } \\
\hline Yes & $218(73)$ & 68 to 78 \\
\hline No & $77(26)$ & 21 to 31 \\
\hline Exempt from review & $5(2)$ & 0.5 to 4 \\
\hline \multicolumn{3}{|c|}{ If ethics review reported, were committees identified? } \\
\hline Yes & $191(88)$ & 83 to 92 \\
\hline No & $27(12)$ & 8 to17 \\
\hline \multicolumn{3}{|c|}{ No of committees, if identified $(n=191)$} \\
\hline $\begin{array}{l}\text { Median (interquartile } \\
\text { range) }\end{array}$ & $1(1-2)$ & NA \\
\hline Range & $1-38$ & NA \\
\hline \multicolumn{3}{|c|}{ If exempt from review, was reason given? } \\
\hline Yes & 2 & NA \\
\hline No & 3 & NA \\
\hline
\end{tabular}


Table $3 \mid$ Characteristics associated with reporting of research ethics review in cluster randomised trials. Values are numbers (row percentages) unless stated otherwise

\begin{tabular}{|c|c|c|c|}
\hline Characteristic & $\begin{array}{l}\text { Ethics review } \\
\text { reported }(n=218)\end{array}$ & $\begin{array}{l}\text { Ethics review not } \\
\text { reported }(n=77)\end{array}$ & $P$ value \\
\hline \multicolumn{3}{|l|}{ Year of publication: } & \multirow{6}{*}{$<0.001$} \\
\hline $2000-1$ & $23(52)$ & $21(48)$ & \\
\hline $2002-3$ & $34(69)$ & $15(31)$ & \\
\hline $2004-5$ & $61(73)$ & $23(27)$ & \\
\hline $2006-7$ & $80(82)$ & $17(18)$ & \\
\hline 2008 & $20(95)$ & $1(5)$ & \\
\hline \multicolumn{3}{|l|}{ Country of study recruitment: } & \multirow{6}{*}{0.623} \\
\hline United States & $84(74)$ & $29(26)$ & \\
\hline UK or Ireland & $37(76)$ & $12(24)$ & \\
\hline Canada & $14(88)$ & $2(13)$ & \\
\hline Australia or New Zealand & $11(79)$ & $3(21)$ & \\
\hline Other & $72(70)$ & $31(30)$ & \\
\hline \multicolumn{3}{|l|}{ Country's level of development: } & \multirow{3}{*}{0.080} \\
\hline Advanced economies & $180(72)$ & $70(28)$ & \\
\hline Emerging and developing economies & $38(84)$ & $7(16)$ & \\
\hline \multicolumn{3}{|l|}{ Unit of randomisation: } & \multirow{3}{*}{0.031} \\
\hline Healthcare organisation & $130(79)$ & $35(21)$ & \\
\hline Non-healthcare organisation & $88(68)$ & $42(32)$ & \\
\hline \multicolumn{3}{|l|}{ Target of interventions: } & \multirow{4}{*}{0.668} \\
\hline Cluster level only & $68(72)$ & $27(28)$ & \\
\hline Individual level only & $80(77)$ & $24(23)$ & \\
\hline Both cluster level and individual level & $70(73)$ & $26(27)$ & \\
\hline \multicolumn{3}{|l|}{ Types of data collection interventions: } & \multirow{5}{*}{0.008} \\
\hline Individual level only & $151(79)$ & $40(21)$ & \\
\hline Cluster level only & $14(64)$ & $8(36)$ & \\
\hline Both individual level and cluster level & $30(75)$ & $10(25)$ & \\
\hline Routine data only & $23(55)$ & $19(45)$ & \\
\hline
\end{tabular}

Sample size calculation accounted for intracluster correlation coefficient:

$\begin{array}{lcc}\text { Yes } & 85(87) & 13(13)\end{array}<0.001$

Analysis accounted for intracluster correlation coefficient:

\begin{tabular}{lccr}
\cline { 1 - 3 } Yes & $161(79)$ & $44(21)$ & \multirow{2}{*}{0.006} \\
\cline { 1 - 3 } No & $57(63)$ & $33(37)$ & \multirow{2}{*}{0.001} \\
\cline { 1 - 2 } $\begin{array}{l}\text { Median (interquartile range) journal impact factor } \\
(\mathrm{n}=289)\end{array}$ & $3.4(2.2-7.3)$ & $2.3(1.7-3.6)$ &
\end{tabular}

dual level in approximately one third of trials, exclusively at the cluster level in another third, and at both levels in the remaining third. In most trials (64\%), data collection interventions were targeted solely at the individual level; 13\% included data collection interventions targeted at participants at both individual and cluster levels, and 8\% collected data exclusively from participants at the level of the cluster. Fifteen per cent had data collection interventions at neither individual nor cluster level, using routinely available data only.

Table 2 shows details of the reporting of research ethics review: 26\% (95\% confidence interval 21\% to $31 \%$ ) of trials failed to report ethics approval, and $73 \%(68 \%$ to $78 \%)$ of trials reported that one or more research ethics committees had reviewed the study.
Among the five trials that reported exemption from ethics review, only two gave explicit reasons for the exemption, both relating to the trials being quality improvement initiatives: "With the primary focus of the project being process quality improvement, none of the mammography centers deemed it necessary to submit the project through its institutional review board process" and "An application for ethical approval was submitted to the [name deleted] Research Ethics Committee. We were informed that the study should be regarded as auditing the effects of change in the hospital's services and research ethics approval was not required for this." Among trials that identified ethics approval, 88\% named the ethics committee(s) involved.

Table 3 presents the results of our analyses investigating characteristics associated with the reporting of ethics review. We excluded the trials that reported exemption from ethics review for the purpose of doing tests of association, as the number of trials in this category was very small $(n=5)$. The frequency of reporting of research ethics review increased strongly over time: in 2000-1, slightly more than half of trials reported ethics approval; this increased to $82 \%$ of trials published in 2006-7 and 95\% of trials published in 2008 (test for trend, $\mathrm{P}<0.001$ ). We found no statistically significant differences according to the country in which the study took place or its level of economic development. Reporting of research ethics approval did not vary significantly by type of randomisation unit or target of planned interventions but was significantly associated with the types of data collection interventions $(\mathrm{P}=0.008)$. Specifically, the empirical estimate of the propensity to report ethics approval for trials using routine data only was 55\% compared with 79\% when data collection was exclusively at the individual level. We found a significant association between reporting of ethics approval and indicators of methodological quality: trials with clearly reported sample size calculations $(\mathrm{P}<0.001)$ and analyses accounting for clustering $(\mathrm{P}=0.006)$ were more likely to report ethics approval. Moreover, the median journal impact factor among trials that reported ethics approval was higher than among those that did not report this approval $(\mathrm{P}<0.001)$.

Table 4 shows details of informed consent procedures reported. Among the 300 trials included in our review, $64 \%(58 \%$ to $69 \%)$ reported on consent, whereas $31 \%$ (26\% to $36 \%$ ) failed to report any consent. Among the trials that reported consent, 17\% reported on consent from more than one type of participantfor example, gatekeeper or participant at cluster level or individual level. Among all 300 trials, 5\% reported consent from gatekeepers, $11 \%$ reported consent from cluster level participants, and 59\% reported consent from individual level participants. Among 16 trials that reported a waiver of consent, only five provided a reason for the waiver: "Outcome data were routinely collected at hospitals and no personal identifiers were transmitted;" "Due to the design of the study, it was not possible to obtain informed consent from the 
$\overline{\text { Table } 4 \mid \text { Reporting of informed consent procedures in cluster }}$ randomised trials $(n=300)$

\begin{tabular}{|c|c|c|}
\hline & Frequency (\%) & $95 \%$ confidence interval of $\%$ \\
\hline \multicolumn{3}{|c|}{ Any consent reported? } \\
\hline Yes & $191(64)$ & 58 to 69 \\
\hline No & $93(31)$ & 26 to 36 \\
\hline Waiver of consent & $16(5)$ & 3 to 8 \\
\hline \multicolumn{3}{|c|}{ Levels of consent, if reported } \\
\hline Multiple levels & $33(17)$ & 12 to 23 \\
\hline Single level & $158(83)$ & 77 to 88 \\
\hline \multicolumn{3}{|c|}{ Any consent reported from gatekeeper? } \\
\hline Yes & $15(5)$ & 3 to 8 \\
\hline No & $285(95)$ & 93 to 98 \\
\hline
\end{tabular}

Any consent reported from participants at cluster level?

\begin{tabular}{lcc}
\hline Yes & $34(11)$ & 8 to 15 \\
\hline No & $266(88)$ & 85 to 92
\end{tabular}

Any consent reported from participants at individual level?

\begin{tabular}{lll}
\hline Yes & $178(59)$ & 54 to 65 \\
\hline No & $122(41)$ & 35 to 47
\end{tabular}

If waiver of consent reported, was justification given?

\begin{tabular}{lcl}
\hline No & $11(69)$ & 41 to 89 \\
\hline Yes & $5(31)$ & 11 to 59 \\
\hline
\end{tabular}

In statement of consent at cluster level, was method reported?

\begin{tabular}{lll}
\hline Yes & $18(53)$ & 35 to 70 \\
\hline No & $16(47)$ & 30 to 65 \\
\hline
\end{tabular}

In statement of consent at individual level, was method reported?

\begin{tabular}{lcc}
\hline Yes & $123(69)$ & 62 to 76 \\
\hline No & $55(31)$ & 24 to 38
\end{tabular}

participants in the trial;" "Data were collected ... as part of the hospital's quality improvement activities and did not require informed consent from the patients;" "The intervention was a policy change at the hospital level, control hospitals were not to change their usual practices, and no individual data was to be collected;" and "Consent was not sought because this study was a pragmatic implementation of health-service provision strategies."

Table 5 shows the results of our analyses investigating characteristics associated with the reporting of consent. We excluded trials that reported waivers of consent for the purpose of doing tests of association, as the number of trials in this category was small $(\mathrm{n}=16)$. We found a strong increase over time in the reporting of consent, from only $36 \%$ of trials in 2000 1 reporting any consent to $80 \%$ of trials in 2006-7 and $95 \%$ of trials in 2008 (test for trend, $\mathrm{P}<0.001$ ). Reporting of consent did not vary significantly by country, level of economic development, or type of cluster. Reporting of consent was significantly associated with the target of planned interventions $(\mathrm{P}<0.001)$ : the empirical estimate of the propensity to report consent for trials with interventions targeting individuals only was $87 \%$ compared with $48 \%$ for trials with interventions targeted solely at the cluster level. Reporting of consent was also significantly associated with types of data collection interventions $(\mathrm{P}<0.001)$ : the empirical estimate of the propensity to report consent for trials collecting routine data only was $29 \%$ compared with $78 \%$ and $40 \%$ for trials with data collection interventions solely at the individual level or the cluster level. We found no significant associations with indicators of methodological quality, although we found a trend towards a significant association with journal impact factor $(\mathrm{P}=0.010)$.

\section{DISCUSSION}

Among 300 cluster randomised trials published between 2000 to 2008 in 150 different journals, 26\% lacked a statement of ethics approval and $31 \%$ failed to report informed consent, but we found evidence of improvement over time. Trials with interventions and data collection activities targeted at the individual level, as well as trials that adhered to important methodological recommendations for cluster randomised trials and those reported in higher impact factor journals, were more likely to report on these basic ethical requirements.

\section{Strengths and limitations of study}

This is the first study to use a large sample of reports from a wide range of journals in health research to formally investigate reporting of ethics practices in cluster randomised trials. As our search strategy had a sensitivity of $90 \%$, we expect our random sample to be representative of most cluster randomised trials in health research published in Medline between 2000 and 2008 ; however, approximately $10 \%$ of cluster randomised trials would not have been identified by our search strategy. Moreover, we cannot completely rule out selection bias in the identification of published reports from the search strategy. Identification of trials as cluster randomised in published reports was pooronly $48.3 \%$ of trials were clearly identified, in contravention of the CONSORT recommendation that trials should be clearly identified as "cluster randomised" in titles or abstracts. To reduce the risk of selection bias, we downloaded the full article, where necessary, to definitively rule out cluster randomisation and proceeded with screening only after two reviewers reached satisfactory agreement in the identification of trials. Nevertheless, some poorly reported trials could have been missed during the screening process. If these trials were also less likely to report on ethical requirements, our results could have overestimated the proportions of trials reporting ethical requirements. To reduce the risk of misclassification of ethics reporting practices and trials' characteristics, we used a consensus between two reviewers who independently extracted information about ethical and methodological factors from the published reports.

\section{Comparison with other studies}

The proportion of cluster randomised trials failing to report on ethical requirements seems to be higher than in non-cluster randomised trials. For example, a review of 259 phase 3 trials of cancer treatments published between 1999 and 2001 in 10 international journals found that $33 \%$ failed to report ethics review and 9\% 
Table $5 \mid$ Characteristics associated with reporting of any informed consent procedures in cluster randomised trials. Values are numbers (row percentages) unless stated otherwise

\begin{tabular}{|c|c|c|c|}
\hline Characteristic & $\begin{array}{l}\text { Consent reported } \\
\qquad(n=191)\end{array}$ & $\begin{array}{l}\text { Consent not reported } \\
\qquad(n=93)\end{array}$ & $P$ value \\
\hline \multicolumn{3}{|l|}{ Year of publication: } & \multirow{6}{*}{$<0.001$} \\
\hline $2000-1$ & $15(36)$ & $27(64)$ & \\
\hline $2002-3$ & $28(57)$ & $21(43)$ & \\
\hline $2004-5$ & $56(69)$ & $25(31)$ & \\
\hline 2006-7 & $74(80)$ & $19(20)$ & \\
\hline 2008 & $18(95)$ & $1(5)$ & \\
\hline \multicolumn{3}{|l|}{ Country of study recruitment: } & \multirow{6}{*}{0.365} \\
\hline United States & $70(65)$ & $38(35)$ & \\
\hline UK or Ireland & $33(67)$ & $16(33)$ & \\
\hline Canada & $14(88)$ & $2(13)$ & \\
\hline Australia or New Zealand & $11(79)$ & $3(21)$ & \\
\hline Other & $63(65)$ & $34(35)$ & \\
\hline \multicolumn{3}{|l|}{ Country's level of development: } & \multirow{3}{*}{0.383} \\
\hline Advanced economies & $161(66)$ & $82(34)$ & \\
\hline Emerging and developing economies & $30(73)$ & $11(27)$ & \\
\hline \multicolumn{3}{|l|}{ Unit of randomisation: } & \multirow{3}{*}{0.036} \\
\hline Healthcare organisation & $98(62)$ & $60(38)$ & \\
\hline Non-healthcare organisation & $93(74)$ & $33(26)$ & \\
\hline \multicolumn{3}{|l|}{ Target of planned interventions: } & \multirow{4}{*}{$<0.001$} \\
\hline Cluster level only & $41(48)$ & $45(52)$ & \\
\hline Individual level only & $90(87)$ & $14(13)$ & \\
\hline Both cluster level and individual level & $60(64)$ & $34(36)$ & \\
\hline \multicolumn{3}{|l|}{ Types of data collection interventions: } & \multirow{5}{*}{$<0.001$} \\
\hline Individual level only & $146(78)$ & $42(22)$ & \\
\hline Cluster level only & $8(40)$ & $12(60)$ & \\
\hline Both individual level and cluster level & $26(69)$ & $12(32)$ & \\
\hline Routine data only & $11(29)$ & $27(71)$ & \\
\hline
\end{tabular}

Sample size calculation accounted for intracluster correlation coefficient:

\begin{tabular}{lcc} 
Yes & 0.142 \\
\cline { 1 - 2 } No & $68(73)$ & $25(27)$ \\
\cline { 1 - 2 }
\end{tabular}

Analysis accounted for intracluster correlation coefficient:

\begin{tabular}{lccc}
\hline Yes & $136(69)$ & $60(31)$ & \multirow{2}{*}{0.253} \\
\cline { 1 - 3 } No & $55(63)$ & $33(38)$ & \\
\hline $\begin{array}{l}\text { Median (interquartile range) journal impact factor } \\
(n=278)\end{array}$ & $3.1(2.2-5.1)$ & $2.6(1.8-4.8)$ & 0.010 \\
\hline
\end{tabular}

failed to report informed consent ${ }^{17}$; the corresponding proportions of cluster randomised trials between 2000 and 2001 in our review were $48 \%$ and $64 \%$. Another review of all 1133 clinical research articles published between 2005 and 2006 in four major clinical journals found that $3.2 \%$ lacked a statement of ethics approval and 5.5\% lacked a statement of informed consent ${ }^{18}$; over the corresponding time period in our review, the proportions were $26 \%$ and $24 \%$. Although higher proportions may be expected given the broader sample of journals included in our review, this is unlikely to be the sole explanation for this discrepancy.

Several possible explanations exist for incomplete reporting of ethics practices in cluster randomised trials. One explanation is uncertainty among investigators about the need to seek research ethics approval and informed consent in different types of cluster randomised trials. A second explanation is that ethics approval or informed consent was in fact not needed (but the authors did not consider this an important detail to report, and journals did not explicitly require waivers to be reported). Despite the ICMJE's recommendations, considerable variability remains in requirements by different journals: some require that ethics approval is stated in the methods section of the manuscript, whereas others require only a separate signed statement from the author; others simply state that studies must have ethics approval but do not mention a method of documentation. ${ }^{19}$ Yet another possibility is misclassification by reviewers, due to poor reporting of consent practices in the trial report (for example, informed consent may have been sought but the authors simply reported that participants "agreed to participate" without an explicit statement of consent). To classify a trial as reporting consent, we required a clear statement of consent in the published report. We were guided by the basic components of informed consent as stated by the Declaration of Helsinki, including that the participants must be adequately informed about the research study's aims, procedures, potential benefits, and risks and the fact that participation is voluntary. ${ }^{9} \mathrm{We}$ found an indication that authors used more rigorous standards when reporting consent practices at the individual level than at the cluster level: $69 \%$ of trials that reported consent at the individual level reported whether the method was written or verbal, but only $53 \%$ of trials that reported consent at the cluster level provided such details. Authors may, therefore, have considered a statement of "agreement" as adequate when describing consent practices from health professionals or other participants at the cluster level but used more conventional descriptors when reporting consent practices at the level of the individual patient. Other reviews of ethics reporting practices have found that failure to disclose ethics approval or informed consent is significantly associated with indicators of lower methodological and reporting quality. ${ }^{1720}$ Here, we found a significant association between reporting of research ethics review and two key indicators of methodological and reporting quality in cluster randomised trials - namely, accounting for clustering in sample size calculations and analyses. Trials that adhered to more rigorous methodological and reporting standards were more likely to report ethics approval. We also found that articles published in journals with higher impact factors were more likely to disclose ethics approval, although we recognise that the impact factor may be an imperfect surrogate for methodological and reporting quality.

Other authors have shown empirically that characteristics of the interventions in cluster randomised trials are associated with consent practices. ${ }^{2122}$ For example, in a review of 152 published cluster randomised trials carried out in primary care settings between 1997 and 2000, Eldridge et al found that the proportions that obtained patients' consent varied between $31 \%$ and $80 \%$, depending on the kinds of 


\section{WHAT IS ALREADY KNOWN ON THIS TOPIC}

Two minimum ethical criteria for randomised controlled trials are approval by a research ethics committee and informed consent from study participants

Cluster randomised trials may have multiple kinds of participants who receive different interventions in the same study

No comprehensive guidelines exist for the ethical conduct and reporting of cluster randomised trials

\section{WHAT THIS STUDY ADDS}

A large proportion of cluster randomised trials are published without disclosure of research ethics approval or informed consent from study participants, but this has improved over time

Failure to disclose ethical protections is associated with characteristics of cluster randomised trials, as well as quality of methods and reporting

Greater clarity in the reporting of ethical issues in cluster randomised trials, including whether consent was sought, from whom, and what consent was for, is recommended

interventions under study. ${ }^{21}$ Although these authors found no evidence that consent practices have changed over time, our results show that the frequencies of reporting research ethics approval and informed consent have increased significantly over time. This may be in response to a small number of publications that have started discussing ethical challenges in cluster randomised trials, such as the UK Medical Research Council's guidelines for cluster randomised trials, published in November 2002, which included a section on ethical factors. ${ }^{10}$ It may also be an indirect result of general increased awareness of the importance of reporting quality brought about by publication of the CONSORT statement and its extension to cluster randomised trials. ${ }^{14}$

\section{Conclusions and policy implications}

Cluster randomised trials are challenging to do and report, not only because they usually involve complex interventions but also because no explicit guidelines with international reach exist to answer basic questions such as from whom, when, and how informed consent ought to be sought. ${ }^{8}$ In the same trial, different components of an intervention may be targeted at multiple kinds of participants, and each kind of participant may need to be approached for informed consent. Moreover, consent may involve consent to be randomised, to receive an intervention, or simply to provide data. We have focused here on the reporting of ethics review and informed consent in cluster randomised trials. Whether current practices are satisfactory in respecting the interests of individual participants in trials depends on the findings of further normative reflection. Conceptual work is needed to determine under what circumstances participants in cluster randomised trials need to be considered research subjects, and then under what circumstances consent for participation is needed. ${ }^{8}$ Greater clarity in the reporting of ethics practices in cluster randomised trials is an important first step in reinforcing good practices in trial conduct and ethics review, for remediation of errors in consent practices and ethics review, and for the development of regulatory guidance for cluster randomised trials. As a minimum, we recommend that authors report the following. (1) Whether research ethics approval was obtained; if ethics review was not required, a reason should be given in the report. (2) Whether informed consent was sought; if a waiver of consent was obtained, this should be stated, together with a reason for the waiver. (3) From whom consent was sought and what consent was for-for example, randomisation, intervention, or data collection.

Contributors: MT, CW, JMG, JCB, MPE, AD, RS, ADMCR, and RFB contributed to the conception and design of the study. MT, ADMcR, CB, SD, JT, and ZS abstracted the data. MT drafted the article and analysed the data. All authors contributed to the interpretation of the data, commented on the first draft and revised the article critically for important intellectual content, and approved the final version. All authors had full access to all of the data (including statistical reports and tables) in the study and can take responsibility for the integrity of the data and the accuracy of the data analysis. MT is the guarantor.

Funding: This study has been funded by operating grants MOP85066 and MOP89790 from the Canadian Institutes of Health Research. The funding agency had no role in the study design; collection, analysis, or interpretation of data; writing of the manuscript; or the decision to submit the manuscript for publication.

Competing interests: All authors have completed the Unified Competing Interest form at www.icmje.org/coi_disclosure.pdf (available on reques from the corresponding author) and declare: no support from any organisation for the submitted work; no financial relationships with any organisations that might have an interest in the submitted work in the previous three years; no other relationships or activities that could appear to have influenced the submitted work

Ethics approval: This study was not approved by a research ethics committee, nor did we request informed consent from the authors of the articles included in our review, because our study did not meet the criteria for human subjects research.

ADMcR holds a fellowship award from the Canadian Institutes of Health Research. JMG and CW both hold Canada Research chairs.

Data sharing: Technical appendix, statistical code, and dataset available from the corresponding author at mtaljaard@ohri.ca.

1 Donner A, Klar N. Design and analysis of cluster randomization trials in health research. Arnold, 2000.

2 Hutton LJL. Are distinctive ethical principles required for cluster randomized controlled trials? Stat Med 2001;20:473-88.

3 Hutton JL, Eccles MP, Grimshaw JM. Ethical issues in implementation research: a discussion of the problems in achieving informed consent. Implement Sci 2008;3:52.

4 Edwards SJL, Braunholtz DA, Lilford RJ, Stevens AJ. Ethical issues in the design and conduct of cluster randomised controlled trials. $B M$ 1999;318:1407-9.

5 Donner A, Klar N. Pitfalls of and controversies in cluster randomization trials. Am J Public Health 2004;94:416-22.

6 Althabe F, Buekens P, Bergel E, Belizán JM, Campbell MK, Moss N, et al. A behavioral intervention to improve obstetrical care. N Engl J Med 2008;358:1929-40.

7 Taljaard M, Weijer C Grimshaw JM, Belle Brown J, Binik A, Boruch R, et al. Ethical and policy issues in cluster randomized trials: rationale and design of a mixed methods research study. Trials 2009;10:61.

8 Weijer C, Grimshaw J, Taljaard M, Binik A, Boruch R, Brehaut J, et al. Ethical challenges posed by cluster randomized trials in health research. Trials 2011;12:100.

9 World Medical Association. Declaration of Helsinki-ethical principles for medical research involving human subjects. 2011. www.wma.net/en/30publications/10policies/b3/index.html.

10 Medical Research Council. Cluster randomized trials: methodological and ethical considerations. 2002. www.mrc.ac.uk/ consumption/idcplg?

IdcService $=$ GET FILE $\&$ dID $=8241 \&$ dDocName $=$ MRC002406\&allowln terrupt $=1$.

11 International Committee of Medical Journal Editors. Uniform requirements for manuscripts submitted to biomedical journals. 2010. www.icmje.org/ethical_6protection.html.

12 Taljaard M, McGowan J, Grimshaw JM, Brehaut JC, McRae A, Eccles MP, et al. Electronic search strategies to identify reports of cluster randomized trials in MEDLINE: low precision will improve with 
adherence to reporting standards. BMC Med Res Methodol 2010;10:15.

13 International Monetary Fund. World economic and financial surveys world economic outlook database. April 2008 edition. 2008. www. imf.org/external/pubs/ft/weo/2008/01/weodata/index.aspx.

14 Campbell MK, Elbourne DR, Altman DG. CONSORT statement: extension to cluster randomised trials. BMJ2004;328:702-8.

15 SCImago Journal and Country Rank. Science analysis. www. scimagojr.com.

16 Falagas ME, Kouranos VD, Arencibia-Jorge R, Karageorgopoulos DE. Comparison of SCImago journal rank indicator with journal impact factor. FASEB J 2008;22:2623-8.

17 Tuech JJ, Pessaux P, Moutel G, Thoma V, Schraub S, Herve CJ. Methodological quality and reporting of ethical requirements in phase III cancer trials. Med Ethics 2005;31:251-5.
18 Finlay KA, Fernandez CV. Failure to report and provide commentary on research ethics board approval and informed consent in medical journals. J Med Ethics 2008;34:761-4.

19 Amdur RJ, Biddle C. Institutional review board approval and publication of human research results. JAMA 1997;277:909-14.

20 Ruiz-Canela M, de Irala-Estevez J, Martinez-Gonzalez MA, Gomez-Gracia E, Fernandez-Crehuet J. Methodological quality and reporting of ethical requirements in clinical trials. J Med Ethics 2001;27:172-6.

21 Eldridge SM, Ashby D, Feder GS. Informed patient consent to participation in cluster randomized trials: an empirical exploration of trials in primary care. Clin Trials 2005;2:91-8.

22 DiGuiseppi C, Coupland C. The design and use of cluster randomised controlled trials in evaluating injury prevention interventions: part 1. Rationale, design and informed consent. Inj Prev 2010;16:61-7.

Accepted: 08 March 2011 